Running Head: ADULT DEVELOPMENTAL TRAJECTORIES

\title{
Adult developmental trajectories of pseudoneglect in the tactile, visual and auditory modalities and the influence of starting position and stimulus length.
}

\author{
Joanna L. Brooks ${ }^{1,2}$, Stephen Darling $*^{3}$. Catia Malvaso ${ }^{2}$, Sergio Della Sala ${ }^{4,5}$
}

${ }^{1}$ Centre for Research on Ageing, Health and Wellbeing, Australian National University, Australia

${ }^{2}$ School of Psychology, University of Adelaide, South Australia

${ }^{3}$ Psychology and Sociology, School of Arts and Social Sciences, Queen Margaret University,

UK

${ }^{4}$ Human Cognitive Neuroscience, Psychology, University of Edinburgh, UK

${ }^{5}$ Centre of Cognitive Ageing and Cognitive Epidemiology, Psychology, University of

Edinburgh, UK

* Corresponding author

Correspondence concerning this article should be addressed to Stephen Darling, Division of Psychology and Sociology, Queen Margaret University, Edinburgh EH21 6UU, UK. Tel 0131 4740000 


\begin{abstract}
Pseudoneglect is a tendency to pay more attention to the left side of space, typically demonstrated on tasks like visuo-spatial line bisection, tactile rod bisection and the mental representation of numbers. The developmental trajectory of this bias on these three tasks is not fully understood. In the current study younger participants aged between 18 and 40 years of age and older participants aged between 55 and 90 years conducted three spatial tasks: 1) visuospatial line bisection - participants were asked to bisect visually presented lines of different lengths at the perceived midpoint; 2) touch-driven tactile rod bisection in the absence of vision - participants were asked to feel the length of a wooden rod with their index finger and bisect the rod at the perceived centre; and 3) mental number line bisection in the absence of vision - participants were asked to listen to a pair of numbers and respond with the numerical midpoint between the pair. The results showed that both younger and older participants demonstrated pseudoneglect (leftward biases) in the visual, tactile and mental number line tasks and that the magnitude of pseudoneglect for each group was influenced by physical or mentally represented starting side (start left versus start right) and stimulus length. We provide an exploration of pseudoneglect in younger and older adults in different tasks that vary in the degree to which mental representations are accessed and argue that pseudoneglect is a result of a right hemisphere attentional orienting process that is retained throughout adulthood. Our results indicate that, contrary to some current models of cognitive ageing, asymmetrical patterns of hemispheric activity may occur in older age.
\end{abstract}

Keywords: pseudoneglect; attention; spatial; ageing. mental number line 
Running Head: ADULT DEVELOPMENTAL TRAJECTORIES

\section{Adult developmental trajectories of pseudoneglect in the tactile, visual and auditory modalities and the influence of starting position and stimulus length.}

\section{Introduction}

Healthy young adults often pay more attention to the left-hand side of space when making spatial judgements, a bias known as pseudoneglect (Bowers \& Heilman, 1980) which also occurs for mental representation. One key class of task on which pseudoneglect is observed are bisection tasks, in which a stimulus is presented and the participant is asked to identify the middle point of the stimulus. Pseudoneglect is consistently demonstrated on three main types of bisection tasks. Visuo-spatial line bisection involves centrally bisecting visually presented horizontal lines of different lengths (Benwell, Harvey \& Thut, 2014a; for review see Jewell \& McCourt, 2000), tactile rod bisection involves centrally bisecting wooden rods of different lengths using touch alone in the absence of vision (Baek, Lee, Kwon et al., 2002; Brooks, Della Sala \& Logie, 2011; Hach \& Schütz-Bosbach, 2012), and mental number line bisection involves mentally representing two or more numbers and bisecting the numerical distance between them (Loftus, Nicholls, Mattingley \& Bradshaw, 2008; Loftus, Nicholls, Mattingley, Chapman \& Bradshaw, 2009; Longo \& Lourenco, 2007; 2010; see also Hubbard, Piazza, Pinel \& Dehaene, 2005). The magnitude of pseudoneglect has been found to be similar, at least for young adults, on visuo-spatial line bisection and mental number line bisection (Longo \& Lourenco, 2007). The observation of pseudoneglect across different modalities is suggestive that a multimodal cognitive mechanism underlies the phenomenon.

The most widely accepted theory of pseudoneglect is that the right cerebral hemisphere orients attention towards contralateral left space due to its dominant role in spatial processing (Heilman \& Van Den Abell, 1979; Reuter-Lorenz, Kinsbourne \& Moscovitch, 1990). This 'Attentional Orienting Hypothesis' is very well supported by behavioural data (Bultitude \& 
Davies, 2006; Toba, Cavanagh \& Bartolomeo, 2011), neuroimaging data (Thiebaut de Schotten, Urbanski, Duffau et al., 2005; Thiebaut de Schotten, Dell'Acqua, Frokel et al., 2011; Varnava, Dervinis \& Chambers, 2013) and clinical data - patients with right hemisphere damage err rightward not leftward on spatial tasks (Dormal, Schuller, Nihoul \& Pesenti \& Andres, 2014; Manfredini, Mancini, Posteraro \& Savazzi, 2013; Robertson \& Marshall, 1993).

The adult developmental trajectory of pseudoneglect, and hence attentional orienting, is a topic of current debate. There is no current agreement about whether or not leftward biases on spatial tasks like visuo-spatial line bisection, tactile rod bisection and mental number line bisection are observed across the full adult lifespan - though there are hints in the data. Jewell and McCourt (2000) noted that healthy older adults show rightward not leftward spatial biases in older age, though this conclusion was based on just two empirical studies that were available at that time (Fujii, Fukatsu, Yamadori \& Kimura, 1995; Stam \& Bakker, 1990). There is now additional research to draw upon. Reduced or reversed rightward biases have been reported on visuo-spatial line bisection or landmark tasks (where participants classify pre-bisected line stimuli: Barrett \& Craver-Lemley, 2008; Benwell, Thut, Grant \& Harvey, 2014b; Schmitz \& Peigneux, 2011; Goedert, LeBlanc, Tsai \& Barrett, 2010; see also Schmitz, Dehon \& Peigneux, 2013). There is also evidence that males and females may produce differential patterns of pseudoneglect over aging, with older females exhibiting more consistent left biases, whilst older males may exhibit a drift into rightward bias with age (Varnava \& Halligan, 2007; Chen, Goedert, Murray, Kelly, Ahmeti, Barrett, 2011). Meanwhile there is evidence for reduced lateral bias in older participants in lateralised visual detection tasks (Learmonth, Thut, Benwell \& Harvey, 2015; Nagamatsu, Carolan, Liu-Ambrose \& Handy, 2011; Nagamatsu, Munkacsy, Liu-Ambrose \& Handy, 2013).

Despite the foregoing, pseudoneglect has also been observed in both younger and older adults on visuo-spatial line bisection (Failla, Sheppard \& Bradshaw, 2003) - though it is to be 


\section{Running Head: ADULT DEVELOPMENTAL TRAJECTORIES}

noted that older adults, unlike younger adults, made errors in the direction of the hand used in bisection). Evidence of pseudoneglect on bisection tasks in older adults was also present in the study of Varnava and Halligan (2007) collapsing across gender, though line-length effects did interact with age.

Pseudoneglect has also been observed in older and younger adults in tactile rod bisection (Brooks, Della Sala \& Logie,2011a) and when recalling details from mentally represented real world scenes (McGeorge, Beschin, Colnaghi, Rusconi \& Della Sala, 2007). Additionally, neurologically intact control participants over the age of 60 years often produce leftward errors on tasks of a spatial nature (see Brooks, Della Sala \& Darling, 2014). Thus there is inconsistency in the literature on visual line bisection and aging, meanwhile although the relationship between aging and tactile and representational pseudoneglect is perhaps less unclear, only very limited systematic research has been carried out in well-matched tasks.

Assuming that pseudoneglect reflects the activity of lateralised cognitive processes, the observation of pseudoneglect in older adults challenges influential models of cognitive ageing. The Hemispheric Asymmetry Reduction in Older Adults (HAROLD) proposes that as the brain ages bilateral recruitment of the two hemispheres increases, especially in prefrontal cortex (Cabeza, 2002; Cabeza, Anderson, Locantore \& McIntosh, 2002; Cabeza, Grady, Nyberg, McIntosh, Tulving \& Kapur 1997; see also Collins \& Mohr, 2013; McGregor, Craggs, Benjamin, Crosson \& White, 2009; Przbyla, Haaland, Bagesteiro \& Sainburg, 2011; Toepper, Markowitsch, Gebhardt et al., 2014). HAROLD is consistent with recent evidence of agerelated changes in attentional control in the left hemifield (Nagamatsu et al., 2011; Learmonth et al., 2015). With regard to pseudoneglect, the HAROLD model essentially predicts that cerebral asymmetry would decline over aging, and consequently so should lateral biases like pseudoneglect that may result from lateralised cognitive processing. The extent to which this attenuation of pseudoneglect should occur is also likely to reflect the degree of of prefrontal 


\section{Running Head: ADULT DEVELOPMENTAL TRAJECTORIES}

involvement - hence tasks that have a high level cognitive component such as representational tasks are likely to be more affected than more perceptual tasks that are likely to be processed by more posterior brain regions. One alternative to HAROLD is the right hemi-ageing model of cognitive ageing which posits that the right hemisphere ages in a different way to the left hemisphere (for discussion see Dolcos, Rice \& Cabeza, 2002; Prodan, Orbelo \& Ross, 2007). Assuming that representational (compared to perceptual) processes engage cognitive networks involved in visual memory to a greater degree, and that these networks are represented to a substantive degree in frontal cortex (see e.g. Courtney, Petit, Maisog, Ungerleider \& Haxby, 1998), the right hemi-ageing approach makes fewer clear differential predictions between representational and perceptual pseudoneglect, as it emphasises specific decreases in frontal asymmetry less than does the HAROLD model.

The CRUNCH hypothesis (Compensation Related Utilisation of Neural Circuits Hypothesis: Reuter-Lorenz \& Cappell, 2008) proposes that more difficult tasks will recruit more cerebral activations, irrespective of brain side, and that on top of this, the level of difficulty at which these additional resources may be brought into use is likely to be lower in older adults. This pattern is actually consistent with the observation of HAROLD effects, if the assumption is made that the most likely areas to be recruited in difficult tasks would be functionally and anatomically corresponding contralateral regions (Berlingeri, Danelli, Bottini, Sberna \& Palesu, 2012). However, the CRUNCH approach is less specific as to patterns of brain activation in ageing, and hence can accommodate preserved lateral biases more easily.

Given the potential challenge to the HAROLD model of the observation of maintained pseudoneglect in older participants, the study reported in this paper aimed to investigate pseudoneglect in older adults across three different bisection tasks. We invited 60 older adults aged 55 to 90 years and 60 younger adults aged 18-40 to conduct three tasks: visuo-spatial line bisection, tactile rod bisection and mental number line bisection. The inclusion of mental 


\section{Running Head: ADULT DEVELOPMENTAL TRAJECTORIES}

number line bisection is of particular significance because there has been no previous investigation of performance on this task in older adults. Mental number line bisection can take the form of reporting a midpoint between two numbers in a pair (Gobel, Calabria, Farne \& Rossetti, 2006) or a making two-alternative forced choice about the numerical position (left or right) of a digit (Loftus et al., 2008). The task devised by Gobel et al. (2006) is more suitable for the current study because the task can be performed in the complete absence of visuospatial processing thus allowing us to explore a purely representational form of pseudoneglect. Representational forms of pseudoneglect, such as are required in the tactile and mental number line tasks, have the potential to be particularly enlightening with regard to the HAROLD hypothesis because they infer the operation of non-perceptual spatial representational or memory systems which are associated with prefrontal cortex (Courtney et al., 1998).

As a secondary aim we also examined the mediation of pseudoneglect across modalities. In line with the previous literature we systematically manipulated lateral starting position and stimulus length for both younger and older adults across task types. For visuospatial bisection starting left has been found to enhance leftward error (Brodie \& Dunn, 2005; Brodie \& Pettigrew, 1996) with greater biases for longer compared to shorter lines (Benwell et al., 2014a; Jewell \& McCourt, 2000; McCourt \& Jewell, 1999) though the definition of 'long' and 'short' depends on the study and different tasks may lead to differential effects (Rueckert, Deravanesian, Baboorian, Lacalamita \& Repplinger, 2002). For tactile rod bisection, in the complete absence of visual input, starting right may boost pseudoneglect (Brooks et al., 2011a) though performance can reflect 'overshooting', where a participant systematically passes the mid-point of the line in the direction of hand travel (so left start leads to right bias and vice versa: Baek et al., 2002) and effects of rod length have been reported by some studies (Laeng, Buchtel \& Butter, 1996; Hach et al., 2011) but not others (Baek et al., 2002; Brooks et al., 2011a). For mental number line bisection, numerical interval (synonymous with line/rod 


\section{Running Head: ADULT DEVELOPMENTAL TRAJECTORIES}

length) significantly affects the degree of bisection error (Gobel et al., 2006; Longo \& Lourenco, 2007) and the presentation of larger numbers first, which are associated with the right-hand side of space, augments the bias (Loftus et al., 2009).

We hypothesised that older adults should display one of three patterns of performance relative to younger adults: 1) less (or reversed) pseudoneglect, especially in tasks which require spatial representation, 2) less (or reversed) pseudoneglect across all tasks, irrespective of representational load or 3) a similar magnitude of pseudoneglect. Prediction (1) is in line with the HAROLD model, prediction (2) with the right hemi-ageing model, whereas (3) would reflect a consistent attentional orientation response across age and is less consistent with either the HAROLD or the right hemi-ageing models. In addition we sought to look at similarities and differences across the three tasks with the aim of understanding $\mathrm{w}$ the detailed nature of pseudoneglect over ageing.

\section{Method}

\subsection{Participants}

The 'younger group' comprised 60 participants aged 18 to 40 years of age $(M=24.00, S D=$ $5.71)$ and the 'older group' 60 participants aged 55 to 90 years $(M=69.77, S D=10.58)$. While gender effects have been reported (Chen, Goedert, Murray, Kelly, Ahmeti \& Barrett, 2011) most studies do not analyse gender or find no effect for adults (as discussed in Jewell \& McCourt, 2000; Brooks et al., 2011a) or find a gender effect as a function of another variable (Hausmann, Ergun, Yazgan \& Gunturkun, 2002). For these reasons, we included both male and female participants in each age group but gender was not systematically balanced. For the older group there were 23 male and 37 female participants. For the younger group there were 12 male and 48 female participants. Young participants were recruited from a university in Scotland and older participants were recruited from a university in South Australia. All 


\section{Running Head: ADULT DEVELOPMENTAL TRAJECTORIES}

participants were right-handed, assessed by asking about their preferred writing hand, reported normal or corrected-to-normal vision and hearing, spoke English as a native language and did not report a history of dyslexia, spatial disorder, dementia, or memory loss. Older participants were screened for mild cognitive impairment with the Mini Mental State Examination (MMSE) directly prior to participating in the current study; a MMSE score of $<27$ indicated cut-off in line with standardised neuropsychological assessment procedures; there were no older adults who fell below this cut-off. To the best of our knowledge, these participants had not previously participated in a study of this nature.

\subsection{Materials}

There were three tasks: visuo-spatial line bisection, tactile rod bisection and mental number line bisection, the materials for each task will be described in turn.

\section{Visuo-spatial line bisection}

Stimuli comprised different length horizontal lines measuring $24 \mathrm{~cm}, 32 \mathrm{~cm}$ and $40 \mathrm{~cm}$ printed in black on A3 paper (lines were $1 \mathrm{~mm}$ thick) by a laser-jet high resolution printer. The length of the lines complemented the lengths of the wooden rods in the tactile rod bisection task. The sheets of paper were white. There was one line printed on A3 strips of paper centrally positioned both horizontally and vertically.

\section{Tactile rod bisection}

A custom-made portable adjustable tactile rod bisection task devised by the authors for a previous study was used here (Figure 1); the design is fully described in a previous paper (Brooks et al., 2011a). There were three wooden doweling rods measuring $24 \mathrm{~cm}, 32 \mathrm{~cm}$, and $40 \mathrm{~cm}$ with rectangular wooden wings attached to the ends of the rods to prevent overshooting 


\section{Running Head: ADULT DEVELOPMENTAL TRAJECTORIES}

during tactile exploration. Hook and loop tape was used to fix the rods to a solid wooden base during tactile exploration; each rod was then easily removed and replaced after each trial.

- Insert Figure 1 about here -

\section{Mental number line bisection task}

We followed the methodology of Gobel et al. (2006) but referenced other studies where applicable (Loftus et al., 2009; Longo \& Lourenco, 2010). Unique aural-verbal number pairs between 111 and 199 were randomly generated using numerical intervals (i.e., stimulus length) of 24 (e.g., 111_ 135), 32 (e.g., 116_148) and 40 (e.g., 127_ 167). There were 30 number pairs in total - 15 with the small number first and 15 with the large number first. The number pairs were pre-recorded in a sound-proof recording booth by the experimenter in order to ensure that all participants listened to exactly the same stimuli. The experimenter was a native English speaker who spoke the number pairs in mono-tone with a $1000 \mathrm{~ms}$ pause between each number in the pair. For the start low condition (equivalent to start left) 15 number pairs were recorded with the small number presented first; for the start high condition 15 number pairs were recorded with the large number presented first as consistent with Loftus et al. (2009) and Longo \& Lourenco (2010). Each number pair was saved into an individual sound file for trial-by-trial presentation (Gobel et al., 2006). A small pilot study was conducted ( $\mathrm{N}=8$ ) with healthy, right handed participants aged between 18 and 38 years in order to test whether or not participants would realise that the same interval was being used for each number pair on every trial. It was confirmed that participants were unaware that the same interval was being used. 


\section{Running Head: ADULT DEVELOPMENTAL TRAJECTORIES}

\subsection{Procedure}

Participants were tested in a quiet room. For younger and older participants the testing suites were similar. The experiment took approximately 45 minutes to complete. The procedure for each task will be described in turn. The three tasks were fully counterbalanced across participants within each age group by starting side and task order. For each task stimulus length was pseudo-randomised - each stimulus length could not be repeated more than twice in a row. A consistent finding is that pseudoneglect does not seem to be driven by any one strategy (Varnava \& Halligan, 2009; Voyer, Saint-Aubin \& Cook, 2014), though time on task may be influential (Benwell, Thut, Learmonth \& Harvey, 2013), so we did not ask participants to use any one strategy with the exception of mental number line bisection (as described below).

\section{Visuo-spatial line bisection}

Participants were seated at a table, positioned so that the centre of their body was aligned with the centre of the table which was covered with a single sheet of white card; when the stimulus was presented it appeared on a uniform white background. The experimenter asked the participant to place their right hand, holding a pen, at a central location (marked by *). Participants were asked to keep their non-dominant hand resting in their lap under the table and to look at a central fixation cross printed on the white card in the middle of the table. The experimenter placed a sheet of A3 paper containing a horizontal line on the table with the centre of the page (and thus the line) aligned with the central fixation cross (marked by + ). There were two conditions under which participants performed visuo-spatial line bisection. In the start left condition participants were required to fixate on the extreme left end-point of the line, hover the pen over the extreme left-hand side of the line, and then bisect the line by marking a vertical line through the perceived centre. In the start right condition this process was reversed (fixate right, hover right, bisect from right). Once completed, each page was 


\section{Running Head: ADULT DEVELOPMENTAL TRAJECTORIES}

removed from the participant's view and replaced with another by the experimenter. There were 30 trials in total: 15 start left trials and 15 start right trials; start side was blocked and counterbalanced across participants and participants were given a short break between startside conditions. Each line length was repeated five times for each start side condition. The experimenter measured the position of the vertical line, the perceived midpoint, relative to the objective midpoint of each line to the closest millimetre.

\section{Tactile rod bisection}

Prior to participation, an index mark (a line drawn by pen) was placed in the middle of the participant's right index finger. Participants were then seated at a table, blindfolded and positioned so that the centre of their body was aligned with the centre of the wooden board. The experimenter guided the participant's right index finger onto the wooden board at a central location (marked by *) which was the baseline position of each trial. Participants were asked to keep their non-dominant hand resting in their lap under the table. In the start left condition the experimenter guided the participant's right index finger from the central baseline position to the extreme left-hand side of the wooden rod; in the start right condition it was the extreme righthand side of the wooden rod. The reaching distance to the rod was approximately $30 \mathrm{~cm}$ and the middle of the rod was centrally aligned with the participant's body centre and the wooden base. When starting and bisecting from the left the participant moved their index finger along the entire length of the rod from left to right, then from right to left, then moved their finger back to the perceived centre of the rod. When starting and bisecting from the right the participant moved their index finger along the entire length of the rod from right to left, then from left to right, then back to the perceived centre. Participants were therefore given one complete scan of the rod before bisection. The participant was required to leave their index finger at the perceived centre until directed by the experimenter who measured the position of the subjective 


\section{Running Head: ADULT DEVELOPMENTAL TRAJECTORIES}

midpoint relative to the objective middle of each rod in terms of direction (left or right) and magnitude (bias to the nearest $\mathrm{mm}$ ). On trials when the index finger was aligned with the objective middle of the rod this was recorded as 'zero bias'. The experimenter recorded the measurement on paper. The experimenter then guided the participant's index finger back to baseline position, changed the rod (randomised order) and began the next trial by guiding the participant's right index finger back to the left or right end-point of the rod. There were 30 trials in total: 15 start left trials and 15 start right trials; start side was blocked and counterbalanced across participants and participants were given a short break between startside conditions (after 15 trials). Participants were allowed to open their eyes as the stimuli were hidden a large cloth. Each rod length was repeated five times for each start side condition.

\section{Mental number line bisection}

The participant was seated and blindfolded; a pre-recorded aural-verbal number pair (e.g., 11_47) was presented to participants binaurally over a pair of speakers; one speaker was positioned to the participant's left-hand side and one speaker to the participant's right-hand side. Immediately after listening to the number pair the participant was required to verbally respond with the perceived numerical midpoint between the two numbers. Participants were explicitly instructed not to calculate (i.e., Gobel et al., 2006; Loftus et al., 2009) and to give the first number that came to mind. On certain trials the participant asked the experimenter to repeat the number pair; the number pair could be repeated once after which, if a further repetition was required, the trial was temporarily abandoned and repeated at the very end of the stimulus set. Before the experimental trials began the speaker volume was adjusted to a comfortable level. Participants completed a practice trial. The perceived midpoint was recorded by the experimenter on paper. Small number first (start left) and large number first (start right) was blocked and counterbalanced across participants and participants were given a short break 


\section{Running Head: ADULT DEVELOPMENTAL TRAJECTORIES}

between start-side conditions. Each numerical length was repeated five times for each start side condition.

\section{Results}

\subsection{Method of Analysis}

The data for 60 participants in the younger group and 60 participants in the older group was analysed for tactile rod bisection, visuo-spatial line bisection, and mental number line bisection. Start side (left versus right) and stimulus length were within-subject variables. Age group was a between-subject variable.

The dependent variable analysis for visuo-spatial and tactile rod bisection expressed the magnitude of absolute bias as a percentage of stimulus length, yielding a percent deviation score; this is a standard method of computing bisection performance for visuo-spatial line bisection and tactile rod bisection (Failla et al., 2003; Hausmann et al., 2002; Baek et al., 2002; Brooks et al., 2011a); a bias of $50 \mathrm{~mm}$ would be proportionally greater for a $240 \mathrm{~mm}$ rod compared to a $400 \mathrm{~mm}$ rod. Negative percent deviation scores indicate a leftward bias while positive values indicate a rightward bias relative to the true centre of the line or the rod. A score of zero reflects no bias. For each task, in line with the previous literature, mean percent deviation was explored both overall, and as a function of starting side and stimulus length using a repeated-measures ANOVA; age (younger, older) was also examined as a between-subject variable. In the mental number line bisection task that required participants to provide a midpoint between two values, the dependent variable was similarly a percentage of deviation from the arithmetical midpoint. The true midpoint of the numeric interval was subtracted from the subjective midpoint to produce an absolute arithmetical bias score, which was then expressed as a percentage of the magnitude of the actual interval. Negative bias indicates a bias to values below (arguably 'left') of the true midpoint while positive values indicate a bias to 


\section{Running Head: ADULT DEVELOPMENTAL TRAJECTORIES}

values above ('right' of) the true midpoint. In all three bisection tasks, a score of zero reflects no bias. In all 3 tasks, the dependent variable reflects a proportion of line or interval length.

One participant represented an extreme left-biased outlier (more than 6 standard deviations to the left of the group mean) on the mental number line task and was excluded from the analyses reported below.

Figure 2 displays the mean percentage deviation for the tasks, and Figure 3 shows the main effects of stimulus length and starting side on the three tasks.

- Figure 2 about here -

- Figure 3 about here -

\subsection{Visuo-spatial line bisection}

For visuo-spatial line bisection there was no effect of age group (younger versus older) on mean percentage deviation $(t(117)=-.671, d=0.12, \mathrm{p}=.503)$. This indicates that the degree of pseudoneglect observed on visuo-spatial line bisection did not differ significantly between younger and older adults. The mean percent deviation for the older participants $(M=-0.69, S D$ $=1.42)$ was significantly different from zero $(t(58)=-3.743, d=0.488, p<.001)$. For the younger participants the mean percent deviation $(M=-.87, S D=1.46)$ was also significantly different from zero $(t(59)=-4.619, d=0.595, \mathrm{p}<.001)$. Figure 4 displays the mean percent deviation scores for each group as a function of start side (start left vs. start right) and stimulus length $(24 \mathrm{~cm}, 32 \mathrm{~cm}, 40 \mathrm{~cm})$ for visuo-spatial line bisection. For older participants there was a significant main effect of start side $\left(F(1,58)=25.170, M S E=4.543, \eta_{p}^{2}=.303, p<.001\right)$, with greater left deviation when starting on the left; a significant effect of stimulus length $(F(2,116)$ 
$=10.072, M_{S E} E^{1}=2.493, \eta_{p}^{2}=.148, p=.001$ ) with longer lines having significantly less leftward deviation than medium $(\mathrm{p}<.001)$ or short $(\mathrm{p}=.003)$, but with no difference between medium and short lines $(\mathrm{p}=.729) .^{2}$ There was no significant interaction between start side and stimulus length $\left(F(2,116)=.114, M S E^{l}=1.288, \eta_{p}^{2}=.002, p=.835\right)$. For younger participants there was a significant main effect of start side $\left(F(1,59)=23.441, M S E=4.242, \eta_{p}^{2}=.284 p<\right.$ $.001)$, a significant effect of stimulus length $\left(F(2,118)=12.791, M S E^{1}=1.152, \eta_{p}^{2}=.178 p<\right.$ $.001)$, and a significant interaction between start side and stimulus length $(F(2,118)=7.183$, $\left.M_{S E} E^{1}=1.392, \eta_{p}^{2}=.109 p=.004\right)$ Post-hoc analysis demonstrated that midpoints for short ( $\mathrm{p}<.001)$ and medium $(\mathrm{p}<.001)$ lines were significantly to the left of those for long lines when the start side was left, whilst there was insufficient statistical evidence to identify a difference between the short and medium lines $(\mathrm{p}=.050)$. Neanwhile,there were no differences according to line length when start side was right (all $\mathrm{p}>.125$ ).

- Insert Figure 4 about here -

\subsection{Tactile rod bisection}

For tactile rod bisection there was no effect of age group (younger versus older) on mean percentage deviation $(t(117)=.143, d=.026, p=.887)$. This indicates that a similar degree of pseudoneglect was observed on tactile rod bisection in both younger and older adults. The mean percent deviation for the older participants $(M=-1.948, S D=3.23)$ was significantly different from zero $(t(58)=-4.628, d=0.603, p<.001)$. For the younger participants the mean percent deviation $(M=-1.87, S D=2.30)$ was significantly different from zero $(t(59)=-6.306$, $d=0.81, p<.001)$. Figure 5 displays the mean percent deviation for each age group as a

\footnotetext{
${ }^{1}$ Due to violation of the assumption of sphericity, the Greenhouse-Geisser correction was applied, $M S E$ and $p$ are reported accordingly.

${ }^{2}$ All post-hoc test p-values reported herein are unadjusted for multiple comparisons. Where statistical significance is asserted the conclusion accounts for multiple comparisons using Bonferroni's method.
} 


\section{Running Head: ADULT DEVELOPMENTAL TRAJECTORIES}

function of start side (start left vs. start right) and stimulus length $(24 \mathrm{~cm}, 32 \mathrm{~cm}, 40 \mathrm{~cm})$. For older participants there was a significant main effect of start side $(F(1,58)=37.283, M S E=$ $\left.79.895, \eta_{p}^{2}=.391, p<.001\right)$ : participants starting on the right showed a greater bias to the left; a significant effect of stimulus length $\left(F(2,116)=5.971, M S E=4.319, \eta_{p}^{2}=.093, p=.003\right)$ : longer $(\mathrm{p}=.021)$ and medium $(\mathrm{p}=.001)$ rods showed significantly more leftward deviation that short, but there was no difference in deviation between longer and medium lines $(\mathrm{p}=.508)$. There was no significant interaction between start side and stimulus length $(F(2,116)=.094$, $\left.M S E^{1}=6.912, \eta_{p}^{2}=.002, p=.910\right)$. For younger participants there was a significant main effect of start side $\left(F(1,59)=21.205, M S E=68.420, \eta_{p}^{2}=.264, p<.001\right)$, a significant effect of stimulus length $\left(F(2,118)=4.764, M S E=5.152, \eta_{p}^{2}=.075, p=.010\right)$, and a significant interaction between start side and stimulus length $\left(F(2,118)=3.731, M S E^{1}=9.009, \eta_{p}^{2}=.059\right.$ $p=.039)$. Post-hoc analysis of this interaction demonstrated no significant differences between midpoints in the start left trials (all $\mathrm{p}>.855$ ), but participants marked the midpoints of medium lines significantly to the left of those for short lines when starting on the right $(\mathrm{p}<.001)$. Although long lines were also marked to the left of short, neither of the comparisons to long lines achieved significance (short $\mathrm{v}$. long $\mathrm{p}=.057$, medium $\mathrm{v}$. long $\mathrm{p}=.077$ ).

- Insert Figure 5 about here-

\subsection{Mental number line bisection}

For mental number line bisection there was no effect of age group (younger versus older) on percentage deviation $(t(117)=-.676, d=0.124, p=.500)$. Mean percentage deviation for the older participants $(M=-4.86, S D=6.86)$ was significantly different from zero $(\mathrm{t}(58)=-5.437$, $d=0.71, p<.001)$. For the younger participants the percentage bias $(M=5.57, S D=4.45)$ was significantly different from zero $(t(59)=-9.700, d=1.252, p<.001)$. The above results indicate a leftward bias on mental number line bisection for both younger and older adults. 


\section{Running Head: ADULT DEVELOPMENTAL TRAJECTORIES}

Figure 6 displays the mean numerical bias scores for each group as a function of first item (low vs. start high) and interval $(24,32,40)$. For older participants there was no main effect of first item $\left(F(1,58)=2.086, M S E^{l}=111.863, \eta_{p}^{2}=.035, p=.154\right)$, a significant effect of interval $\left(F(2,116)=5.609, M S E^{l}=92.656, \eta_{p}^{2}=.088 p=.007\right)$. Post-hoc analysis demonstrated a significant difference between medium and both short and long intervals, with medium intervals having greater deviation towards the low 'left' end of the scale than short intervals ( $p$ $=.013)$ and long intervals $(\mathrm{p}<.001)$. Deviations for long intervals did not differ significantly to short $(\mathrm{p}=.975)$ intervals. The interaction between first item and interval was not significant, $\left(F(2,116)=2.876, M S E^{l}=123.332, \eta_{p}^{2}=.047, p=.074\right)$. For younger participants there was a significant main effect of first item $\left(F(1,59)=6.898, M S E=69.677, \eta_{p}^{2}=.105, p=.011\right)$; bisections erred further to the left (low) end of the scale when the first digit was high (right). There was no significant effect of interval $\left(F(2,118)=2.318, M S E=52.190, \eta_{p}^{2}=.038, p=\right.$ .103); but there was a significant interaction between first item and interval $(F(2,118)=15.729$, $\left.M S E=33.934, \eta_{p}^{2}=.210, p<.001\right)$ driven by a tendency to respond with lower values in the start high condition - especially for longer relative to shorter intervals, a pattern confirmed by post-hoc analyses that identified significantly lower responses for start-high trials versus startlow trials in medium $(\mathrm{p}<.001)$ intervals. There was a similar pattern with lower responses for start-high trials in large intervals $(\mathrm{p}=.018)$, but this comparison was not significant following adjustment (Bonferroni) for multiple comparisons. There was no significant difference for short lines $(\mathrm{p}=.176)$. Interestingly, a broadly similar pattern, though not forming a significant interaction, was observed in the older adults, as can be seen in Figure 6.

- Insert Figure 6 about here-

\subsection{Analyses across tasks}


A repeated measures ANOVA with task as a within-subject variable (x3) and age group as a between-subject variable ( $\mathrm{x} 2$ ) was conducted using percentage bias scores. There was a significant effect of task $\left(F(2,234)=45.160, M S E^{1}=19.410, \eta_{p}^{2}=.278, p<.001\right)$ but no significant interaction between age group and task $\left(F(2,234)=.345, M S E^{1}=19.410, \eta_{p}^{2}=.003\right.$, $p=.638)$. There was no main effect of age group $\left(F(1,117)=0.436, M S E=15.267, \eta_{p}^{2}=.004\right.$, $p=.511$ ). Post-hoc analyses were conducted for task (with biases collapsed for younger and older adults). There were significant differences in all comparisons reflecting the fact that leftward biases were greater for tactile rod bisection compared to visuo-spatial line bisection and for mental number line bisection than for both tactile rod bisection and visual line bisection, clearly visible in Figure 2 (all $\mathrm{p}<.001)$.

In order to explore the possibility that age effects influenced lateral bias within the recruited age groups, two simple regression analyses were run, one for older and one for younger adults. Percentage deviations on the three experimental tasks were used as continuous predictors to generate linear regression models predicting age $\mathrm{e}^{3}$. Age was selected as the target because this would enable all three bisection tasks to be simultaneously be entered as predictors and hence assessed for their independent contribution to lateral bias. Gender was also entered, so that effects of gender could be controlled for. Among the young adults, the only significant predictor of age was mental number line bisection - the leftward lateral bias in line bisection in younger adults declined for older members of the younger adult sample. There were no other significant predictors of age. A similar analysis was conducted in the older adults. Here, the only significant predictor of age was gender (female participants tended to be older), though we note that visual line bisection tended towards significance $(\mathrm{p}=.071)$. An omnibus regression analysis across all participants assessing the same predictors of age failed to generate a

\footnotetext{
${ }^{3}$ Age data was missing for one participant in the young condition
} 


\section{Running Head: ADULT DEVELOPMENTAL TRAJECTORIES}

significant model. Specific details of the regression models are shown in Table 1, alongside the direct correlation of each task with age.

-Insert Table 1 about here

- Insert Figure 7 about here-

Finally, there was no significant correlation between bias for visuo-spatial and tactile rod bisection (Pearson's $r(119)=-.105, p=.257$ : Spearman's $R=-.085, p=.357$ ); there was no correlation between visuo-spatial and mental number line bisection bias $(r(119)=.060, p=$ $.516: R=.086, p=.354)$; and there was no correlation between on tactile rod and mental number line bisection $(r(119)=.074, p=.421 R=.011, p=.904)$. Figure 7 illustrates the relationships between the three different bisection task biases and age.

\subsection{Power to Detect Null Effects}

Whilst we found no significant effects of age on pseudoneglect, our statistical analysis was not powered to detect small effects, hence the possibility that our null effects overlooked small effects of age should be quantified. The present study was sufficiently powered to identify large effects $(d>.80)$ with a power $>.95$. This is the magnitude of effects observed previously by Brooks, et al. (2011a). Following data collection it was possible to place confidence limits around the observed effect sizes (using the tools described by Wuensch, 2014). In the case of visual and mental number line bisection the upperbound of the interval (0.483 in both cases) approached but did not reach a 'medium' effect size (Cohen, 1992). The tactile rod bisection (upperbound $=0.386$ ) interval fell some way below Cohen's estimated 'medium' effect size. In the omnibus ANOVA analysis across tasks the upperbound on effect size $\left(\eta_{p}^{2}\right)$ of the two non-significant effects $(0.042$ for the main effect of age and 0.039 of the task $\mathrm{x}$ age interaction) did not reach the conventional estimate of a 'medium' effect (Richardson, 2011). Of course, these intervals do not preclude the possibility that relatively 


\section{Running Head: ADULT DEVELOPMENTAL TRAJECTORIES}

small age effects may exist, but they do suggest an absence of large age effects on any of the three tasks used.

\section{Discussion}

The aim of the current study was to conduct a multimodal investigation of pseudoneglect in younger and older adults and to investigate whether or not the magnitude of pseudoneglect was systemically modulated in the same way across modalities. In the current study, older adults aged 55 to 90 years and younger adults aged 18-40 years conducted three tasks: visuo-spatial line bisection, tactile rod bisection, and mental number line bisection. For each task lateral starting side and stimulus length were systematically manipulated. For both younger and older adults pseudoneglect was clearly demonstrated on visuo-spatial line bisection, tactile rod bisection and mental number line bisection - these are novel findings and extend the previous research in this field (e.g. Brooks et al., 2011a; De Agostini et al., 1999; McGeorge et al., 2007; Varnava \& Halligan, 2007).

A number of regression analyses were conducted in order to investigate whether there was any evidence of relationships with age both within and across the sampled age groups. These showed only one significant relationship: older young adults bisected further to the right than younger ones in the mental number line task, suggesting a weakening of left-lateral mental number line biases with age in younger adults. However, although apparent in our data for young adults, a similar or extrapolated pattern was not seen in the older adults (who showed no relationship between age and lateral bias) or in omnibus analysis across the two age groups. There was no robust evidence of any similar pattern on tactile bisection or on visual line bisection. Some care is thus required in interpreting this finding, though one speculative possibility might be that older young adults within a university setting may have been more efficient in utilizing an arithmetic strategy on mental number line bisection due to increasing 


\section{Running Head: ADULT DEVELOPMENTAL TRAJECTORIES}

experience of mathematics use in their studies, though further research is called for to clarify this. The trend towards right bias in visual line bisection (a non-significant association between age and reduced left bias in the older adult sample) is a somewhat equivocal result that contributes to a similarly equivocal literature where some reports indicate a decline in left bias in visual bisection (Barret et al., 2008; Chen et al., 2011) whilst some so do not (Failla et al., 2003; Varnava \& Halligan, 2007; Brooks et al., 2011). We note that the present data form a non-significant trend only apparent in the older adult sample when that sample is considered on its own, and as thus have limited explanatory power. It should be noted that the minimum age of older adult participants in our study is a little younger than in other comparable visual pseudoneglect studies (e.g. Benwell et al, 2014; Goedert et al, 2010; Learmonth et al, 2015; Nagamatsu,et al, 2011; Schmitz \& Peigneux, 2011). These younger participants show a greater left-bias. However, the regression analyses did not furnish substantial evidence of lifetime agedependent influences on pseudoneglect tasks.

Correlations between the three bisection tasks here were surprisingly low, suggesting that the three tasks measure rather different things - this is perhaps unsurprising given the fairly gross differences between the three tasks, and is consistent with the low correlation observed between the landmark task and line bisection (Rueckert et al., 2002). The likely reason for this is that the portion of task performance on the three bisection tasks that is driven by 'pseudoneglect' lateral bias is rather small - again unsurprising given the fact that pseudoneglect biases are almost by definition small. However, these small correlations also imply that there is a good deal of heterogeneity between the three tasks used here, and hence it is possible that the three tasks could be subject to differential patterns of lateral change across ageing. Our data do not reject this possibility and the variation across tasks of effects of start direction and length supports this conclusion too. 


\section{Running Head: ADULT DEVELOPMENTAL TRAJECTORIES}

The present results for visual bisection are at odds with some published data which has suggested that older adults show reduced pseudoneglect (Fujii et al., 1995; Stam \& Bakker, 1990; Benwell et al., 2014b; Schmitz \& Peigneux, 2011; Barrett \& Craver-Lemley, 2008; Goedert et al., 2010), so it is necessary to consider why this may be. One speculative candidate may be method: Benwell et al. (2014b) and Schmitz \& Peigneux (2011) both used variants of the 'Landmark' task, in which judgements are made about a pre-bisected presented line: it may be that this task requires more fine grained perceptual evaluation, and thus elicits higher levels of real or perceived difficulty, , and that this causes additional recruitment of areas in the contralateral hemisphere, which is a clear possibility under the CRUNCH model of aging (Reuter-Lorenz and Cappell, 2008) and may also be so under the HAROLD model (Cabeza, 2002; Cabeza, et al., 1997; 2002). However, the other studies listed have used more traditional line bisection, so this cannot be the sole explanation. Another possibility relates to the gender balance in our study (61\% female in the older adults), given that Varnava and Halligan (2007) and Chen et al. (2011) reported some gender effects on these tasks. However, it is to be noted that both the Varnava and Halligan and also the Failla et al. (2003) studies showed some evidence of left bias in older adults, in contrast to, for example, Goedert et al., 2010, Barrett et al. (2008) and Chen et al. (2011), yet all of these studies utilised groups that approached or attained equality in gender mix despite varying in the extent to which they evidenced line bisection pseudoneglect. Finally, we acknowledge that it is difficult to provide evidence for the absence of any age effect, but our post-hoc power analysis indicates that it is unlikely that we have overlooked medium effects.

Turning to the mental number line and tactile bisection conditions; these showed consistent leftward biases, with no evidence for age differences - a pattern which was associated with a high degree of confidence in ruling out the possibility of a medium effect, and hence, just as for visual bisection in our study, unlikely to result from a Type 2 error. The only 


\section{Running Head: ADULT DEVELOPMENTAL TRAJECTORIES}

published work on aging and tactile bisection (Brooks et al., 2011a) also found no age-related bisection difference, and the present study is the first to report an exploration of mental number line bisection in the context of the magnitude of varied pseudoneglect tasks in older adults. The foregoing discussion raises a difficult question as to why the age-related effect data are inconsistent within the visual bisection literature, but less so in the tactile bisection literature. One possibility, suggested by the fact that age effects are generally observed on landmark tasks and detection tasks but that there is more variability of results on visual line bisection tasks, is that line bisection might have a considerable motor component which remains subject to lateral bias. A related suggestion might be that bias persists on our visual line bisection task in older adults because of functional impairment of the corpus callosum with aging - relevant given that our participants bisected with the dominant right hand. These explanations, however, cannot account for the presence of aging-resistant biases on the MNL, a task with minimal motor involvement, and have difficulty with data indicating that bisection bias can be dissociated from motor responses (MacLeod \& Turnbull, 1999) or be seen in the absence of spatiomotor motor response (McGeorge at al, 2007).

A second, more persuasive, possibility is that higher level representations may remain subject to lateral bias in aging, whilst purely visual perceptual processing may not. The landmark task is a highly perceptual task with limited requirement for representation, although line bisection, whilst undeniably perceptual, arguably has more components requiring representation, such the representation required prior to generating a motor response. The tactile task clearly requires mental representation, given that the participant must establish a spatial representation of the rod from dynamic tactile information, and the evidence of bisection error on the mental number line task also implies that the mathematical interval is interpreted as a spatial representation - the task is clearly, at any rate, not one of visual perception. This interpretation is consistent with the fact that age effects are seen on landmark tasks, on visual 


\section{Running Head: ADULT DEVELOPMENTAL TRAJECTORIES}

line bisection in some studies, but seem to be absent on tactile and MNL bisection. Incidentally, it is possible that the integration of visual bisection in a within subjects design alongside two representational tasks in the present study may have increased participants' tendency to invoke representation when carrying out the task, and hence be related to the relative resistance to agerelated change. Overall, this argument is consistent with the data presented by Barrett and Craver-Lemley (2008) showing the presence of age effects in bisection (older adults showed no bisection bias) alongside an absence of age effects in an implicit lateral bias task (the location of a drawn item relative to a piece of paper) and reduced age effects alongside consistent bias in a spatial-syntactic bias task. An attractive aspect of this explanation is that it is consistent with putative evidence of identifiable differences between perceptual psudoneglect and representational pseudoneglect (Darling, Logie \& Della Sala, 2012; Della Sala, Darling \& Logie, 2010). Irrespective of the specific validity of this hypothesis though, the data presented here taken in association with the literature imply a degree of heterogeneity within the phenomenon of 'pseudoneglect' which needs to be incorporated in future models of attention, representation and aging.

The finding of pseudoneglect across modalities and across lifespan is difficult to accommodate within the popular HAROLD model of cognitive ageing (Cabeza, 2002; Cabeza, et al., 1997; 2002). HAROLD argues for more bilateral activation of the cerebral hemispheres in older age. Originally the model focused on frontal areas, though there is evidence supporting reduction in asymmetry in tasks associated with posterior areas too (Nagamatsu et al., 2011; Learmonth et al., 2015).Our results demonstrating equivalent pseudoneglect in older and younger adults are incompatible with the HAROLD model proposal (see also Toepper et al., 2014). The observation of consistent lateral bias in representational tasks like mental number line and tactile bisection are especially problematic under the HAROLD model with its focus on decreasing hemispheric asymmetry over age especially in prefrontal cortex, given the role 


\section{Running Head: ADULT DEVELOPMENTAL TRAJECTORIES}

prefrontal cortex is known to play in spatial memory (Courtney et al., 1998). For similar reasons, our findings are not in line with the right hemi-ageing model (Dolcos et al., 2002; Prodan et al., 2007) nor with findings indicating rightward biases for older adults (Benwell et al., 2014b).

In contrast to the HAROLD model, our data are indicative of asymmetries in hemispheric processing in the direction of the attentional orienting hypothesis (Heilman \& Van Den Abell, 1979; Reuter Lorenz et al., 1990) and suggest that tasks like visuo-spatial line bisection, tactile rod bisection and mental number line bisection predominantly engage attentional orienting mechanisms in the right parietal cortex (Thiebaut de Schotten et al., 2005; Varnava et al., 2013). We assert, based on the present data, that a very similar neural signature is produced in older adults. In fact, the CRUNCH hypothesis (Reuter-Lorenz \& Cappell, 2008) may have some explanatory power here due to its proposal that extra brain areas may be recruited in response to task demand in aging: in simple perceptual tasks like the landmark task it may be the case that the most appropriate area to recruit in the case of added task demand may be the equivalent contralateral visual processing area, leading to attenuation of bias, whereas in tasks requiring memory the added load may recruit ipsilateral areas associated with spatial memory, higher order function and flexible attention. This is an explanation that could be tested experimentally.

The most fitting account of the data is that attentional orienting led to the left portion of the line or rod being perceived as longer than the right portion; hence the perceived mid-point was shifted leftward in each case (i.e., Bultitude \& Davies, 2006; Toba, Cavanagh \& Bartolomeo, 2011). The same theory may apply for mental number line bisection with the equivalent process being a rounding down of the numerical midpoint (Loftus et al., 2009). Across tasks the left side of the stimulus seemed to be more heavily weighted - perceptually or in the mind's eye - than the right side of the stimulus (Brooks, Logie, McIntosh \& Della Sala, 


\section{Running Head: ADULT DEVELOPMENTAL TRAJECTORIES}

2011b). In older participants this attentional orienting response was clearly maintained to a strong degree, across all thre tasks(Reuter-Lorenz \& Cappell, 2008).

Of critical importance is the fact that across all three tasks lateral starting side and stimulus length were important for modulating the magnitude of pseudoneglect for younger and older adults. Enhanced leftward biases are often observed under conditions that favour the right hemisphere (Brodie \& Pettigrew, 1996; Brodie \& Dunn, 2005); starting left significantly enhanced the magnitude of pseudoneglect and this is consistent with the concept of the right hemisphere receiving an 'activation advantage' when the left portion of the line was viewed first (i.e., Brodie, 2010). Arguably, in the start right condition the left hemisphere received an activational advantage which was balanced by right hemisphere attentional orienting due to the spatial nature of the task - thus attenuating leftward bias in this condition. For tactile rod bisection there was a reversed start right effect which also complements the previous research (Brooks et al., 2011a). During tactile rod bisection a representation of space driven by touch alone is built sequentially, over time; when the exploration and bisection direction was consistent with the direction of attentional orienting (right to left) pseudoneglect was enhanced but reduced when inconsistent (Brooks et al., 2011a). For mental number line bisection we theorised that the start left and start right conditions were synonymous with presenting a small or large number first respectively; in support of this assumption is the finding that pseudoneglect for mental number line bisection - also a task in which stimuli were presented sequentially in the absence of direct visuo-spatial processing - was consistent with that for tactile rod bisection: presenting a larger number first magnified leftward error in younger adults, a finding also stated in the previous literature (Loftus et al., 2009). In support of this argument is the fact that symmetrical biases were not observed when start side was counterbalanced - for any task - which is evidence that the bias was genuinely driven by 


\section{Running Head: ADULT DEVELOPMENTAL TRAJECTORIES}

attentional orienting and not another mechanism. The lack of symmetrical bias suggests that 'overshooting' (Baek et al., 2002) cannot account for the data on its own.

In the current study broadly similar stimulus length effects and start side effects were observed in each task for younger and older adults and this suggests that the same process underlies performance in each age group. The aim of the current study was not to explore line length per se; rather we wished to explore the systematic deviation from midpoint across three tasks focusing on differences between age groups. Our visual bisection results are somewhat inconsistent with the previous literature that suggests the magnitude of pseudoneglect increases with line length: in our study an opposite pattern was seen with larger effects at shorter lengths. However, in comparison to previous research we possibly did not use a range of lines sufficient to demonstrate the typical scaling effect. Visuo-spatial line bisection tasks have adopted different line lengths; lines up to $28 \mathrm{~cm}$ (Rueckert et al., 2002), 20cm (Varnava and Halligan 2007), 16cm (Brodie \& Dunn, 2005); and $25 \mathrm{~cm}$ (calculated from visual angle) (McCourt \& Jewell, 2000). In the current study the lines fell outside these typical ranges because it would not have been suitable to employ a $1 \mathrm{~cm}$ rod, for example, for tactile rod bisection. This difference could potentially be informative - it is possible that visual bisection biases might persist in older adults in situations where the to-be-bisected object is sufficiently long. Our results for tactile and mental number line bisection are broadly in line with previous literature: Laeng et al. (1996) found leftward biases for rods of different lengths (24, 28, 30, 35, and $40 \mathrm{~cm}$ ) and that the bias increased with rod length. Likewise Hach et al. (2011) used rod lengths of $20 \mathrm{~cm}, 30 \mathrm{~cm}$ and $40 \mathrm{~cm}$ - very similar to our study - and found that bias scaled with rod length when the stimuli were centrally presented. For mental number line bisection numerical interval, synonymous with line/rod length significantly affects the degree of bisection error (Gobel et al., 2006; Longo \& Lourenco, 2007) and the presentation of larger numbers first, which are associated with the right-hand side of space, augment the bias (Loftus et al., 2009). 


\section{Running Head: ADULT DEVELOPMENTAL TRAJECTORIES}

Zorzi, Priftis and Umiltà (2002) found, for neglect patients, midpoints were typically reported in the direction of the larger number consistent with a rightward bias that also increased with numerical distance.

Although we found very few significant age effects in this study, in future it would be interesting to conduct a computerised landmark task alongside the tasks used in this study. Our tentative hypothesis regarding the role of representational pseudoneglect generates a clear prediction that older participants would show reduced pseudoneglect on a landmark task than on representational bisection tasks.

In conclusion, the present study has provided evidence that pseudoneglect occurs for both younger and older adults and across modalities, but that there is considerable variability in pseudoneglect across tasks within individual participants. There was minimal evidence for agerelated differences in pseudoneglect. The best account for these data is the attentional orienting hypothesis, and the maintenance of attentional orienting over typical ageing. Moreover, the

results have illustrated that the phenomenon of pseudoneglect is robust, consistent, and call for further empirical investigations to consider systematic manipulations of the bias in participants of different ages. We maintain that modifications to the HAROLD model of ageing are needed in order to provide for instances of pseudoneglect, both perceptual and representational, that indicate asymmetrical activity in older age.

\section{Statistical Tools}

Basic statistical analysis in this project was carried out using SPSS (Version 21: IBM Corp, 2012) and JASP (Love et al., 2015). Power calculations were carried out using G*Power (Faul, Erdfelder, Lang \& Buchner, 2007). 


\section{References}

Baek, M. J., Lee, B. H., Kwon, J. C., Park, J. M., Kang, S. J., Chin, J., et al. (2002). Influence of final search direction on tactile line bisection in normal subjects. Neurology, 58(12), $1833-1838$.

Barrett, A.M., \& Craver-Lemley, C.E. (2008). Is it what you see, or how you say it? Spatial bias in young and aged subjects. Journal of the International Neuropsychological Society, $14(4), 562-70$.

Benwell, C. S. Y., Harvey, M., \& Thut, G. (2014a). On the neural origin of pseudoneglect: EEG correlates of shifts in line bisection performance with manipulation of line length. NeuroImage, 86, 370-380.

Benwell, C., Thut, G., Grant, A. \& Harvey, M. (2014b). A rightward shift in the visuospatial attention vector with healthy aging. Frontiers in Ageing Neuroscience, 6, 113.

Benwell, C., Thut, G., Learmonth, G. \& Harvey, M. (2013). Spatial attention: Differential shifts in pseudoneglect direction with time-on-task and initial bias support the idea of observer subtypes. Neuropsychologia, 51 (13), 2747-2756.

Berlingeri, M., Danelli, L., Bottini, G., Sberna, M. \& Paulesu, E. (2013). Reassessing the HAROLD model: Is the hemispheric asymmetry reduction in older adults a special case of compensatory-related utilization of neural circuits? Experimental Brain Research, 224(3), 393-410.

Bowers, D., \& Heilman, K. M. (1980). Pseudoneglect - effects of hemispace on a tactile line bisection task. Neuropsychologia, 18(4-5), 491-498.

Brooks, J. L., Della Sala, S., \& Logie, R. (2011a). Tactile rod bisection in the absence of visuospatial processing in children, mid-age and older adults. Neuropsychologia, 49(12), $3392-3398$ 
Brooks, J. L., Logie, R. H., McIntosh, R. D., \& Della Sala, S. (2011b). Representational pseudoneglect for an auditory-driven spatial working memory task. Quarterly Journal of Experimental Psychology, 64(11), 2168-2180.

Brooks, J., Della Sala, S., \& Darling, S. (2014). Representational pseudoneglect: A review. Neuropsychology Review, 24, 148-165.

Brodie, E. E. (2010). Reflecting upon line bisection: mirror reversal increases the magnitude of pseudoneglect. Neuropsychologia, 48(5), 1517-1520.

Brodie, E. \& Dunn, E. (2005). Visual line bisection in sinistrals and dextrals as a function of hemispace, hand, and scan direction. Brain \& Cognition, 58, 149-156.

Brodie, E. E., \& Pettigrew, L. E. L. (1996). Is left always right? Directional deviations in visual line bisection as a function of hand and initial scanning direction. Neuropsychologia, 34(5), 467-470.

Bultitude, J. H., \& Davies, A. M. A. (2006). Putting attention on the line: Investigating the activation-orientation hypothesis of pseudoneglect. Neuropsychologia, 44(10), 1849-1858.

Cabeza, R. (2002). Hemispheric asymmetry reduction in older adults: The HAROLD model. Psychology and Aging, 17(1), 85-100.

Cabeza, R., Anderson, N. D., Locantore, J. K., \& McIntosh, A. R. (2002). Aging gracefully: Compensatory brain activity in high-performing older adults. NeuroImage, 17(3), 13941402.

Cabeza, R., Grady, C. L., Nyberg, L., McIntosh, A. R., Tulving, E., Kapur, S., et al. (1997). Age-related differences in neural activity during memory encoding and retrieval: A positron emission tomography study. Journal of Neuroscience, 17(1), 391-400.

Chen, P., Goedert, K. M., Murray, E., Kelly, K., Ahmeti, S., \& Barrett, A. M. (2011). Spatial Bias and Right Hemisphere Function: Sex-Specific Changes with Aging. Journal of the International Neuropsychological Society, 17(3), 455-462. 
Cohen, J. (1992). A power primer. Psychological Bulletin, 112(1), 155-159.

Collins, K., \& Mohr, C. (2013). Performance of younger and older adults in lateralized right and left hemisphere tasks support the HAROLD model. Laterality, 18 (4), 491-512.

Courtney, S.M., Petit, L., Ungerleider, L.G., Maisog, J.M., \& Haxby, J.V.(1998). An Area Specialized for Spatial Working Memory in Human Frontal Cortex. Science, 279(5355), $1347-1351$.

Darling, S., Logie, R.H., Della Sala, S. (2012). Representational pseudoneglect in line bisection. Psychonomic Bulletin \& Review, 19, 879-883.

De Agostini, M., Curt, F., Tzortzis, C., \& Dellatolas, G. (1999). Comparing left and right hand in line bisection at different ages. Developmental Neuropsychology, 15(3), 379-394.

Della Sala, S., Darling, S., Logie, R.H. (2010). Items on the left of memory representations are better remembered. Quarterly Journal of Experimental Psychology, 63, 848-855

Dolcos, F., Rice, H. J., \& Cabeza, R. (2002). Hemispheric asymmetry and aging: right hemisphere decline or asymmetry reduction. Neuroscience and Biobehavioral Reviews, 26(7), 819-825.

Dormal, V., Schuller, A. M., Nihoul, J., Pesenti, M., \& Andres, M. (2014). Causal role of spatial attention in arithmetic problem solving: Evidence from left unilateral neglect. Neuropsychologia, 60, 1-9.

Failla, C. V., Sheppard, D. M., \& Bradshaw, J. L. (2003). Age and responding-hand related changes in performance of neurologically normal subjects on the line-bisection and chimeric-faces tasks. Brain and Cognition, 52(3), 353-363.

Faul, F., Erdfelder, E., Lang, A.-G., \& Buchner, A. (2007). G*Power 3: A flexible statistical power analysis program for the social, behavioral, and biomedical sciences. Behavior Research Methods, 39, 175-191.

Fujii, T., Fukatsu, R., Yamadori, A., \& Kimura, I. (1995). Effect of age on the line bisection 
Running Head: ADULT DEVELOPMENTAL TRAJECTORIES

test. Journal of Clinical and Experimental Neuropsychology, 17(6), 941-944.

Goedert, K.M., Leblanc, A., Tsai, S.W., \& Barrett, A.M. (2010). Asymmetrical effects of adaptation to left- and right-shifting prisms depends on pre-existing attentional biases. Journal of the International Neuropsychology Society, 16(5), 795-804.

Gobel, S. M., Calabria, M., Farne, A., \& Rossetti, Y. (2006). Parietal rTMS distorts the mental number line: simulating 'spatial' neglect in healthy subjects. Neuropsychologia, 44(6), 860868.

Hach, S. \& Schütz-Bosbach, S. (2012). Touching base: The effect of participant and stimulus modulation factors on a haptic line bisection task. Laterality, 17 (2), 180-201.

Hausmann, M., Ergun, G., Yazgan, Y., \& Gunturkun, O. (2002). Sex differences in line bisection as a function of hand. Neuropsychologia, 40(3), 235-240.

Heilman, K. M., \& Van Den Abell, T. (1979). Right hemispheric dominance for mediating cerebral activation. Neuropsychologia, 17(3-4), 315-321.

Hubbard, E. M., Piazza, M., Pinel, P., \& Dehaene, S. (2005). Interactions between number and space in parietal cortex. Nature Reviews Neuroscience, 6(6), 435-448.

IBM Corp. (2012). IBM SPSS Statistics for Windows, Version 21.0. Armonk, NY: IBM Corp.

Jewell, G., \& McCourt, M. E. (2000). Pseudoneglect: A review and meta-analysis of performance factors in line bisection tasks. Neuropsychologia, 38(1), 93-110.

Laeng, B., Buchtel, H. A., \& Butter, C. M. (1996). Tactile rod bisection: hemispheric activation and sex differences. Neuropsychologia, 34(11), 1115-1121.

Learmonth, G., Thut, G., Benwell, C.S., \& Harvey, M. (2015). The implications of statedependent tDCS effects in aging: Behavioural response is determined by baseline performance. Neuropsychologia, 74, 108-119.

Loftus, A. M., Nicholls, M. E. R., Mattingley, J. B., \& Bradshaw, J. L. (2008). Left to right: Representational biases for numbers and the effect of visuomotor adaptation. Cognition, 


\section{Running Head: ADULT DEVELOPMENTAL TRAJECTORIES}

107(3), 1048-1058.

Loftus, A. M., Nicholls, M. E. R., Mattingley, J. B., Chapman, H. L., \& Bradshaw, J. L. (2009). Pseudoneglect for the bisection of mental number lines. Quarterly Journal of Experimental Psychology, 62(5), 925-945.

Longo, M. R., \& Lourenco, S. F. (2007). Spatial attention and the mental number line: Evidence for characteristic biases and compression. Neuropsychologia, 45(7), 1400-1407.

Longo, M. R., \& Lourenco, S. F. (2010). Bisecting the mental number line in near and far space. Brain and Cognition, 72(3), 362-367.

Love, J., Selker, R., Marsman, M., Jamil, T., Dropmann, D., Verhagen, A. J., Ly, A., Gronau, Q. F., Smira, M., Epskamp, S., Matzke, D., Wild, A., Rouder, J. N., Morey, R. D. \& Wagenmakers, E.-J. (2015). JASP (Version 0.7) [Computer software].

Manfredini, A., Mancini, F., Posteraro, L., \& Savazzi, S. (2013). The two sides of spatial representation in neglect patients: The same spatial distortion for different patterns of performance. Neuropsychologia, 51, 1867-1877.

McCourt, M. E., \& Jewell, G. (1999). Visuospatial attention in line bisection: stimulus modulation of pseudoneglect. Neuropsychologia, 37(7), 843-855.

McGeorge, P., Beschin, N., Colnaghi, A., Rusconi, M. L., \& Della Sala, S. (2007). A lateralized bias in mental imagery: Evidence for representational pseudoneglect. Neuroscience Letters, 421(3), 259-263.

McGregor, K. M., Craggs, J. G., Benjamin, M. L., Crosson, B., \& White, K. D. (2009). Agerelated changes in motor control during unimanual movements. Brain Imaging and Behavior, 3(4), 317-331.

McLeod, M.S., \& Turnbull, O.H. (1999). Motor and perceptual factors in pseudoneglect. Neuropsychologia, 37(6), 707-713. 


\section{Running Head: ADULT DEVELOPMENTAL TRAJECTORIES}

Nagamatsu, L.S., Carolan, P., Liu-Ambrose, T.Y., \& Handy, T.C. (2011). Age-related changes in the attentional control of visual cortex: a selective problem in the left visual hemifield. Neuropsychologia, 49(7), 1670-1678.

Nagamatsu, L.S., Munkacsy, M., Liu-Ambrose, T. \& Handy, T.C. (2013). Altered visualspatial attention to task-irrelevant information is associated with falls risk in older adults. Neuropsychologia, 51(14), 3025-3032.

Prodan, C. I., Orbelo, D. M., \& Ross, E. D. (2007). Processing of facial blends of emotion: Support for right hemisphere cognitive aging. Cortex, 43(2), 196-206.

Przybyla, A., Haaland, K. Y., Bagesteiro, L. B., \& Sainburg, R. L. (2011). Motor asymmetry reduction in older adults. Neuroscience Letters, 489(2), 99-104.

Reuter-Lorenz, P.A., \& Cappell, K. A. (2008). Neurocognitive ageing and the compensation hypothesis. Current Directions in Psychological Science, 17, 177-182.

Reuter-Lorenz, P. A., Kinsbourne, M., \& Moscovitch, M. (1990). Hemispheric control of spatial attention. Brain and Cognition, 12(2), 240-266.

Richardson, J.T.E. (2011). Eta squared and partial eta squared as measures of effect size in educational research. Educational Research Review, 6(2), 135-147.

Rueckert, L., Deravanesian, A., Baboorian, D., Lacalamita, A., \& Repplinger, M. (2002). Pseudoneglect and the cross-over effect. Neuropsychologia, 40(2), 162-173.

Robertson, I. H., \& Marshall, J. (1993). Unilateral neglect: Clinical and experimental studies. Hove: Erlbaum

Schmitz, R., Dehon, H., \& Peigneux, P. (2013). Lateralized processing of false memories and pseudoneglect in aging. Cortex, 49 (5), 1314-1324.

Schmitz, R., \& Peigneux, P. (2011). Age-related changes in visual pseudoneglect. Brain and Cognition, 76(3), 382-389.

Stam, C.J. \& Bakker, M. (1990). The prevalence of neglect: superiority of neuropsychological 
Running Head: ADULT DEVELOPMENTAL TRAJECTORIES

over clinical methods of estimation. Vlinical Neurology and Neurosurgery, 92(3), 229-35.

Thiebaut de Schotten, M., Urbanski, M., Duffau, H., Volle, E., Lévy, R., Dubois, P. \& Bartolomeo, P. (2005). Direct evidence for a parietal-frontal pathway subserving spatial awareness in humans. Science, 309 (5744), 2226-2228.

Thiebaut de Schotten, M., Dell'Acqua, F., Frokel, S., Simmons, A., Vergani, F., Murphy, D. \& Catani, M. (2011). A lateralised brain network for visuospatial attention. Nature Neuroscience, 14 (10), 1245-1246.

Toba, M.-N., Cavanagh, P., \& Bartolomeo, P. (2011). Attention biases the perceived midpoint of horizontal lines. Neuropsychologia, 49(2), 238-246.

Toepper, M., Markowitsch, H. J., Gebhardt, H., Beblo, T., Bauer, E., Woermann, F. G., Driessen, M., Sammer, G. (2014). The impact of age on prefrontal cortex integrity during spatial working memory retrieval. Neuropsychologia, 59, 157-168.

Varnava, A., Dervinis, M., \& Chambers, C. (2013). The Predictive Nature of Pseudoneglect for Visual Neglect: Evidence from Parietal Theta Burst Stimulation. PLOS ONE, 8 (6), e65851.

Varnava, A., \& Halligan, P. W. (2007). Influence of age and sex on line bisection: A study of normal performance with implications for visuospatial neglect. Aging Neuropsychology and Cognition, 14(6), 571-585.

Varnava, A., \& Halligan, P. W. (2009). Line bisection: Does introspection inform cognitive strategy? Neuropsychologia, 47(1), 280-283.

Voyer, D., Saint-Aubin, J., \& Cook, C. (2014). Strategies and pseudoneglect on luminance judgments: An eye-tracking investigation. Journal of Experimental Psychology, Human Perception and Performance, 40 (5), 1789-1798.

Wuensch, K. (2014). Tests of equivalence and confidence intervals for effect sizes. Retrieved from http://core.ecu.edu/psyc/WuenschK/docs30/Equivalence-EffectSizeCI.pdf on the 24th of November, 2014. 
Running Head: ADULT DEVELOPMENTAL TRAJECTORIES

Zorzi, M., Priftis, K., \& Umilta, C. (2002). Brain damage - Neglect disrupts the mental number line. Nature, 417(6885), 138-139. 